\title{
Designer non-toxic derivatives dodge resistance
}

\section{ces}

all mice

inoculated with AmB-,

AmBMU- or

AmBAU-

resistant

strains

survived the

infection
The antifungal amphotericin $\mathrm{B}$

(AmB) kills microorganisms

effectively. Moreover, resistance to

$\mathrm{AmB}$ is very rare because the drug

binds to ergosterol - a key lipid

required for fungal survival and virulence - and extracts it from the fungal cell membrane. However, the use of $\mathrm{AmB}$ is limited by side effects such as kidney damage that are due to the off-target binding of the drug to cholesterol in host cells. Now, Davis et al. synthesize new, resistance-evading derivatives of

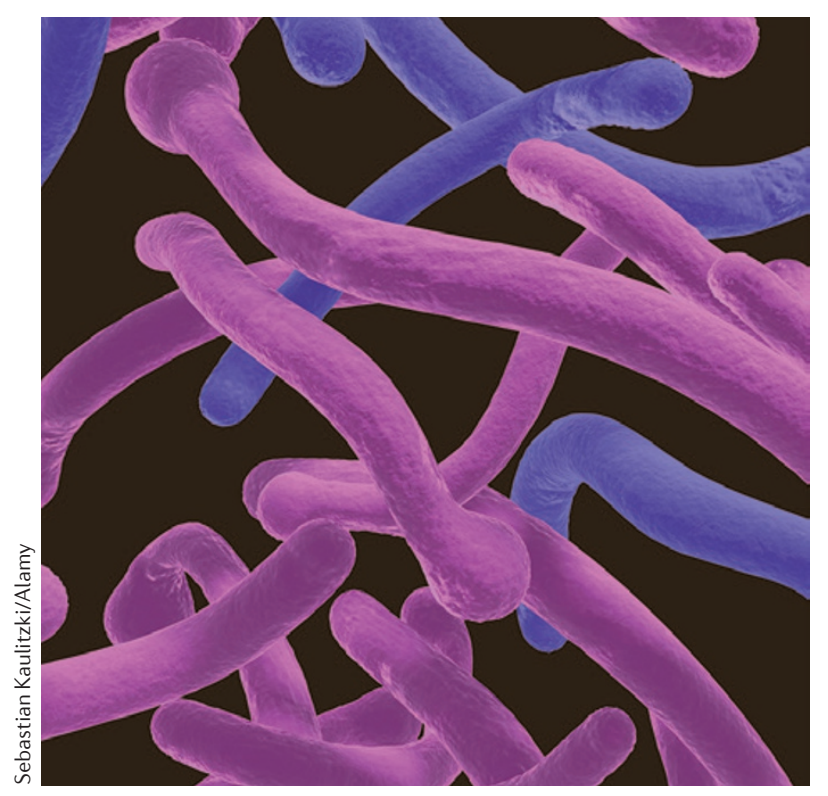

AmB that selectively bind to ergosterol, and show their efficacy against fungal infections in mice.

Previously, many AmB derivatives had been synthesized by altering groups on the C16-C41 bond of the molecule; however, most derivatives retained a carbon-carbon bond at this position. Here, Burke and colleagues reasoned that replacing the C16-C41 bond with a carbonnitrogen bond would eliminate an intramolecular salt bridge between the C41 carboxylate and the mycosamine appendage of $\mathrm{AmB}$, thus perhaps altering the binding of the drug to sterol lipids.

The authors used a three-step reaction sequence to replace the carboxylic acid group on C16 with a urea group to give $\mathrm{AmB}$ methyl urea (AmBMU) or AmB amino urea (AmBAU). Using isothermal titration calorimetry, the authors showed that AmBMU and AmBAU bind to ergosterol, but not to cholesterol, suggesting that these derivatives could be less toxic than AmB but still have antifungal properties.

In a neutropenic mouse model of disseminated candidiasis, AmBMU and AmBAU were considerably more effective than $\mathrm{AmB}$ at reducing the Candida albicans burden in the kidneys 24 hours after treatment, suggesting improved antifungal efficacy. Importantly, these new derivatives proved to be safer than AmB: whereas mice given an acute dose of $4 \mathrm{mg}$ per $\mathrm{kg} \mathrm{AmB}$ all perished, AmBAU induced more than $50 \%$ lethality only at doses of $64 \mathrm{mg}$ per $\mathrm{kg}$, and the same dose of AmBMU did not induce any observable side effects.

The authors next investigated the potential for development of drug resistance to AmBMU and AmBAU. Interestingly, inoculation with wild-type strains of C. albicans was lethal to mice, whereas all mice inoculated with AmB-, AmBMU- or AmBAU-resistant strains survived the infection, suggesting that strains that are resistant to any of these drugs are avirulent.

Overall, this study shows that these new urea-containing derivatives retain the antifungal efficacy and low vulnerability to resistance of AmB but exhibit considerably lower toxicity. Moreover, the authors note that the synthesis of these derivatives is efficient and can be scaled up for production relatively easily.

Natasha Bray

ORIGINAL RESEARCH PAPER Davis, S. A. et al. Nontoxic antimicrobials that evade drug resistance. Nat. Chem. Biol. 11, 481-487 (2015) 